\title{
Effects of Rheological Property of Coating Liquid and Withdrawal Velocity on Dip Coating Process in Manufacturing of Capsules
}

\author{
Kazunori YASUDA ${ }^{* 1}$, Takashi KOSHIBA ${ }^{* 2}$, and Noriyasu MoRI ${ }^{* 1}$ \\ ${ }^{* 1}$ Department of Mechanophysics Engineering, Graduate School of Engineering, Osaka University, \\ 2-1, Yamadaoka, Suita, Osaka 565-0871, Japan \\ *2 Department of Mechanical Engineering, Nara National College of Technology, \\ 22, Yatacho, Yamato-koriyama, Nara 639-1080, Japan
}

(Received : June 9, 2003)

\begin{abstract}
In the present paper, to elucidate effects of the formation and breakage of filament on the dip coating process in the manufacturing of capsules, we measured the filament length between the tip of the coated rod and the liquid surface when the filament broke, and examined the dependence of the filament length on the rheological properties of coating liquids and withdrawal velocity to optimize the dip coating process. The coating liquids were aqueous solutions of syrup as Newtonian liquids and mixture liquids of aqueous solutions of syrup and water-soluble polymer (polyacrylamide) as polymeric liquids.Consequently, it is found that a large withdrawal velocity brings a shorter time at the filament breakage in Newtonian liquids regardless of their shear viscosities. The experimental equation which represents the relation between the breakage length of the filament and the shear viscosity of the coating liquid are presented. In polymeric liquids, a large elongational viscosity causes a large filament length.The large withdrawal velocity was ineffective upon the reduction of the breakage time of the filament. The use of polymeric liquids with large spinnability reduces the efficiency of manufacturing in dip coating process. The normal stress difference of the coating liquid reduces the coating thickness, so that the coating liquid containing polymer is required to have a small spinnability and a moderate normal stress difference.
\end{abstract}

Key Words: Dip coating process / Manufacturing of capsule / Shear viscosity / Elongational viscosity / Filament length

\section{INTRODUCTION}

A coated surface protects the substrate, and provides a good feature and a characteristic function to the substrate surface. For example, in an automobile industry, the coating on the car body is necessary for rust preventive and decoration. On the other hand, the coating process is also used to product parts having a thin thickness. A capsule for medicine is shaped by the coating of a material on the rod-shaped substrate with a hemisphere at the rod tip. After the drying out, the thin film is removed from the substrate surface. In such a process, the capsule for a medicine is manufactured.

There are many methods for coating on a solid surface and the most familiar one is the painting by a brush or a roller. As we know empirically, it is difficult to make a thin liquid layer uniformly on the solid surface by the painting. The simplest

*1 $_{1}$ To whom correspondence should be addressed.

E-mail: yasuda@mech.eng.osaka-u.ac.jp

Tel \& Fax: 06-6879-7309 coating process is dip coating, where a substrate is immersed in a bath containing a coating liquid and is withdrawn from the bath. The advantage of this process is simplicity, low cost and ease of control, so that this process is often used in many industries.

In the dip coating process, the coating thickness is extremely interesting scientifically and industrially. The thickness of the film in the dip coating is determined by various factors - the rheological properties of the coating liquid, the speed of withdrawal, and the angle of the substrate withdrawn from the bath. There has been many previous studies on the theoretical and the experimental analysis in the dip coating. In these works, the researchers focused their attention on a coating thickness.

Ruschak $^{1)}$ provided a good review of coating flows. For a Newtonian liquid, White and Tallmadge ${ }^{2)}$ gave the theoretical relationship for estimating the film thickness on a flat plate. Spiers et al. ${ }^{3)}$ theoretically considered the entrainment of liquid films onto a vertical surface. Marques et al. ${ }^{4}$ used a 
computational approach to determine the velocity profile of fluids in a thin film. Krozel et al. ${ }^{5)}$ observed the transient coating process of dip coating. Weinstein and Ruschak ${ }^{6}$ considered a dip coating on a planar non-vertical substrate. Qu et al. ${ }^{7)}$ experimentally examined the hydrodynamics of dipcoated films of volatile fluids.

The coating liquid is required to have a low viscosity for easy and quick coating under high shear rate, and a high viscosity for good adhesion of the liquid on the surface under low shear rate. To attain such a characteristic, the coating liquid generally contains polymers. The product part having a thin thickness manufactured by the dip coating process, such as capsules for medicine, is made of polymeric materials. Thus, the coating liquid used in the real manufacturing generally has non-Newtonian characteristics. For a nonNewtonian liquid, Tanguy et al. ${ }^{8), 9)}$ numerically studied the dip coating process with a finite element method. Their simulated results of the interface shape of the coating liquid are in good agreement with their experimental ones. Moreover, the normal stress difference of the coating liquid causes a thin thickness of the film. Mori et al. ${ }^{10)}$ investigated the relation between the coating thickness and the rheological property of the coating liquid and reported that the normal stress difference reduced the coating thickness. The rheological property of the coating liquid affects the coating thickness, so that the coating liquid should be prepared carefully and appropriately for a good quality of the product.

On the other hand, the manufacturing efficiency is very important in the real manufacturing process. For the high efficiency, the speed of withdrawal is a key factor. In the process of dip coating, especially for the capsule manufacturing, a filament of the coating liquid is formed between the coating liquid in the bath and the end of the hemisphere when the product is withdrawn from the bath, and before long the filament breaks. The filament stretching and breakage seem to affect the manufacturing efficiency of the capsule. Therefore, it is important to examine the phenomena in the filament stretching and breakage to attain high efficiency in the coating process. Furthermore, the filament stretching has a relation to the rheological property of the coating liquid such as an elongational viscosity. Thus, in the dip coating process, the good quality of the product and the high manufacturing efficiency depend on the rheological properties of the coating liquid.

In the real manufacturing, the most satisfactory conditions for both the rheological property of the coating liquid and the high efficiency of the manufacturing are required. In the present paper, as a first step to elucidate the relation between them in the dip coating process of the capsules, we will measure the critical length of the filament when it breaks, and examine the effect of the rheological property of the coating liquid on the breakage length to optimize the dip coating process.

\section{TEST FLUIDS AND EXPERIMENTAL METHOD}

\subsection{Test fluids and rheology}

Various coating liquids are used for dip coating in manufacturing process. In the present experiment, Newtonian liquids and non-Newtonian liquids were tested. Newtonian liquids are $80 \mathrm{wt} \%, 85 \mathrm{wt} \%, 87 \mathrm{wt} \%, 88 \mathrm{wt} \%$ and $90 \mathrm{wt} \%$ aqueous solutions of syrup. Syrup solutions are easy to control their viscosities by varying the ratio of syrup to water. Furthermore, we used three polymeric solutions containing polyacrylamide (PAA, AH-70P, $M_{\mathrm{v}} \approx 1 \times 10^{7}$, Sanyo Chemical Industries, Ltd, Japan) as non-Newtonian liquids, where the PAA was added in the $85 \mathrm{wt} \%$ aqueous solutions of syrup at 3 ppm, 5 ppm and 7 ppm by weight, respectively. Polyacrylamide solutions have a shear-thinning and a stretch-thickening viscosity, and are easy to handle because of water solubility. Water in these test fluids evaporates quickly because of high concentration of syrup, so that we conserve these fluids carefully to prevent the evaporation.

We measured shear viscosities of the test fluids using an Ubbelohde viscometer for Newtonian liquids and a cone-andplate rheometer (Rheosol-G2000, UBM Co., Ltd, Japan) for non-Newtonian ones at $25^{\circ} \mathrm{C}$, respectively. Figure 1 shows the concentration dependence of viscosity for the aqueous solutions of syrup. It is found that the shear viscosity increases

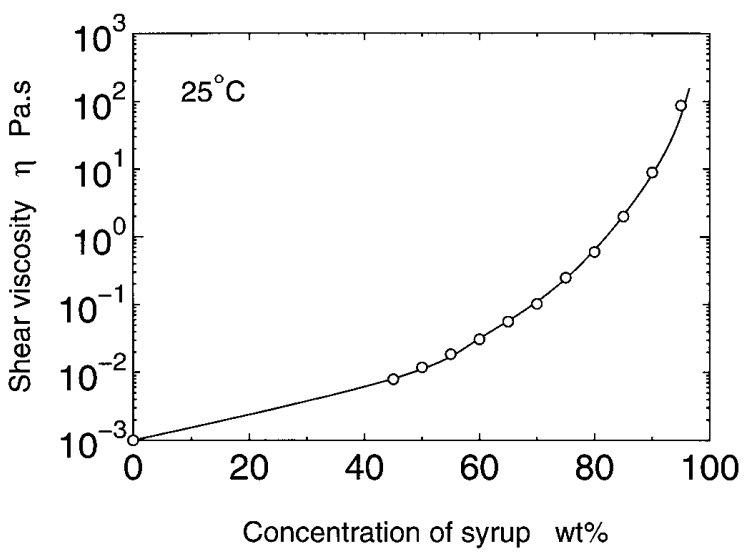

Fig.1 Shear viscosity dependence of aqueous solutions of syrup on concentration of syrup at $25^{\circ} \mathrm{C}$. 
as the concentration of the syrup increases. Figure 2 shows the viscosity for $3 \mathrm{ppm}$ and $7 \mathrm{ppm}$ solutions of PAA in $85 \mathrm{wt} \%$ aqueous solution of syrup. Both the polymeric solutions have shear-thinning viscosities which are considered to be almost the same regardless of the PAA concentration. The viscosity reaches that of the $85 \mathrm{wt} \%$ aqueous solution of syrup, that is 2 Pa.s, at high shear rate.

Furthermore, we have to pay attention to the elongational viscosity of coating liquid when we consider the filament stretching in the dip coating process. Thus, we measured the elongational viscosities of the polymeric liquids using a spinline rheometer. Table I shows the results for $3 \mathrm{ppm}, 5 \mathrm{ppm}$ and $7 \mathrm{ppm}$ solutions of PAA in $85 \mathrm{wt} \%$ aqueous solution of syrup. In general, polymeric liquids exhibit stretch-thickening in the elongational viscosity. However, we were not able to observe a clear stretch-thickening viscosity for the test liquids because it was difficult to vary the elongational rate widely in the spinline rheometer. Thus, the apparent elongational viscosity exhibited a constant value at the elongational rates

Table I Elongational viscosities of $85 \mathrm{wt} \%$ aqueous solutions of syrup containing PAA, with standard deviations.

\begin{tabular}{c|c}
\hline \hline $\begin{array}{c}\text { Concentration of PAA } \\
\text { (ppm by weight) }\end{array}$ & $\begin{array}{c}\text { Elongational viscosity } \\
\text { (Pa.s) }\end{array}$ \\
\hline $0^{*}$ & $6^{* *}$ \\
3 & $50 \pm 6.5$ \\
5 & $52 \pm 8.1$ \\
7 & $70 \pm 10$ \\
\hline
\end{tabular}

* Newtonian liquid

** Theoretical result calculated from shear viscosity

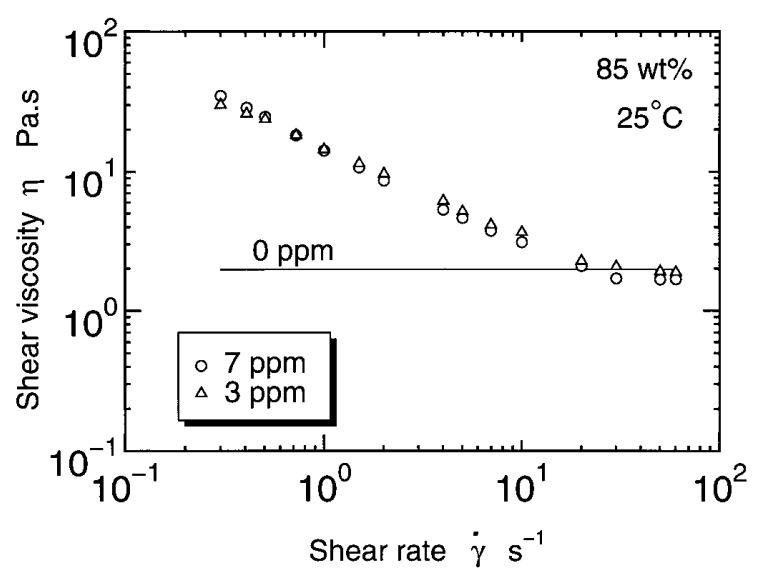

Fig.2 Shear viscosity dependence of polymeric solutions on shear rate in $85 \mathrm{wt} \%$ solutions of syrup containing PAA at the concentration of $3 \mathrm{ppm}$ and $7 \mathrm{ppm}$ at $25^{\circ} \mathrm{C}$, respectively. from $2 \mathrm{~s}^{-1}$ to $20 \mathrm{~s}^{-1}$. These constant values for three polymeric liquids are indicated in Table I. It is found that the elongational viscosity of the polymeric liquid increases with increasing the concentration of PAA. For the $85 \mathrm{wt} \%$ aqueous solution of syrup the elongational viscosity is estimated at $6 \mathrm{~Pa} . \mathrm{s}$ which is equal to three times as large as the shear viscosity.

\subsection{Experimental apparatus and filament length measurement}

In the dip coating process the coating liquid is held in a dip bath and the immersed product is withdrawn from the bath. In the real manufacturing process the dip bath is equipped with a circulation system of the coating liquid, and moreover, the liquid is always refreshed by adding the fresh liquids in the bath. This circulating process prevents the polymeric liquids from degrading and drying at the liquid surface. Following this system, we prepared the experimental apparatus as shown in Fig.3.

The coated rod, which is made of brass, has $6 \mathrm{~mm}$ in diameter and a spherical tip of $3 \mathrm{~mm}$ in radius. This rod is equipped at the linear guide to slide only in the direction of gravity smoothly. We wound a non-stretched wire connected to the rod by a speed-controlled electric motor to withdraw the rod at a constant speed. The vessel made of glass, which is 84 $\mathrm{mm}$ in diameter and $58 \mathrm{~mm}$ in depth, was filled with the test liquid. This diameter of the glass vessel is enough larger than the diameter of the coated rod. To prevent the liquid from drying at the surface of the liquid the fresh liquid was always

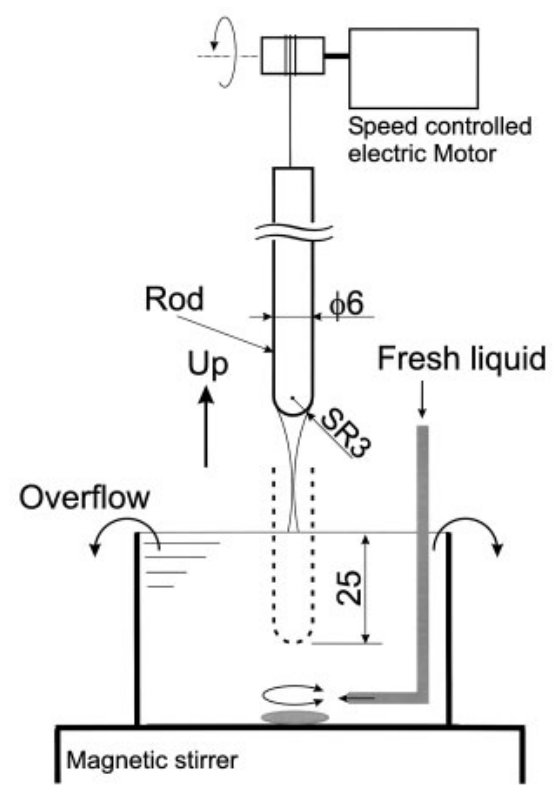

Fig.3 Experimental apparatus for dip coating. 
fed into the bottom of the vessel and overflowed at a flow rate small enough to being only negligible effects on the filament stretching process. Moreover, the test liquid is stirred slowly by a magnetic stirrer placed under the vessel and accurately kept at $25 \pm 1^{\circ} \mathrm{C}$ using a temperature controller during the experiment.

The rod was immersed in the coating liquid through the length of $25 \mathrm{~mm}$ from the tip and withdrawn at a constant velocity in the range of $1.98-7.25 \mathrm{~mm} / \mathrm{s}$. In the preliminary experiment, we confirmed that the immersed length of the rod hardly affect an experimental result described in the next section. In the present work, we repeated the measurement five times in each experimental condition and removed the coating liquid from the rod surface each time using a spatula.

When the rod is withdrawn from the bath, a filament is formed between the tip of the rod and the surface of the coating liquid in the bath as shown in Fig.4. We recorded the formation of the filament with a digital video camera (DCRVX1000, Sony Co.), and measured the breakage length of the filament $L_{\mathrm{B}}$, which is a height from the liquid surface in the vessel to the tip of the rod at the breakage of the filament.

\section{RESULTS AND DISCUSSION}

\subsection{Results for Newtonian liquids}

In this subsection, we will describe the relationship between the breakage length and the withdrawal velocity for the Newtonian liquids. Figure 5 shows the dependence of the breakage length on the withdrawal velocity of the rod in each aqueous solution of syrup. It is found that the breakage length linearly increases with increasing withdrawal velocity. For the liquid having a low shear viscosity the breakage length is

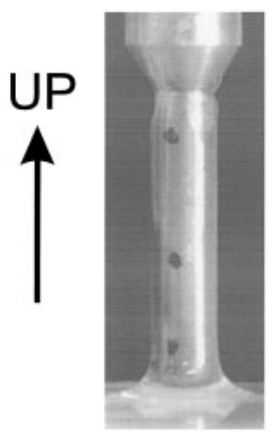

(a)

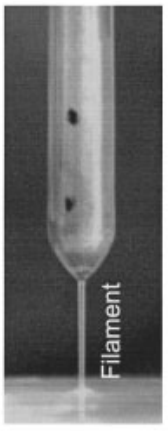

(b)

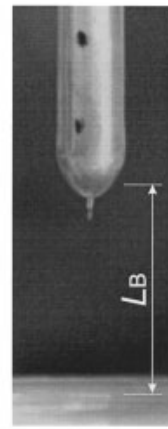

(c)
Fig.4 Dip coating process: (a) the coated rod being withdrawn from the dip bath, (b) a filament formed between the surface of the coating liquid and the rod tip, (c) an instantaneous aspect just after filament breakage. slightly effected by the withdrawal velocity, but it increases drastically as the withdrawal velocity increases for the liquid having a large shear viscosity. In this figure, the breakage length is about $3 \mathrm{~mm}$ in every liquid when the withdrawal velocity reaches zero. This is because the surface tension of the coating liquid and the wetting of the coating liquid on the rod surface cause to form a meniscus between the rod tip and the liquid surface in the bath, and the filament is surely formed between them even if the withdrawal velocity of the rod is very small. Here, we obtained the following equation from the experimental results:

$$
L_{\mathrm{B}}=0.28 \eta V_{\mathrm{w}}+0.0029
$$

where $L_{\mathrm{B}}$ is the breakage length (m), $\eta$ the shear viscosity (Pa.s) and $V_{\mathrm{w}}$ the withdrawal velocity $(\mathrm{m} / \mathrm{s})$. The value of 0.28 is a proportional coefficient having a unit of $\mathrm{Pa}^{-1}$ and the value of 0.0029 the initial length at $V_{\mathrm{w}}=0(\mathrm{~m} / \mathrm{s})$ depending on various factors, e.g., the surface tension of the coating liquid, the wetting of the coating liquid on the rod surface, and the shape of the rod tip. Therefore, in general, we can obtain the simple equation:

$$
L_{\mathrm{B}}=C \eta V_{\mathrm{w}}+L_{\mathrm{B}}^{\prime}
$$

where $C$ is a proportional coefficient and $L_{\mathrm{B}}^{\prime}$ an initial breakage length. The solid lines in Fig.5 show the numerical results calculated from Eq.(1). It is found that Eq.(1) can represent the experimental resuls well.

Figure 6 shows that the relationship between the shear viscosities and the breakage lengths. The breakage lengths linearly increase with increasing shear viscosity at a fixed withdrawal velocity. In this figure, the breakage length is not

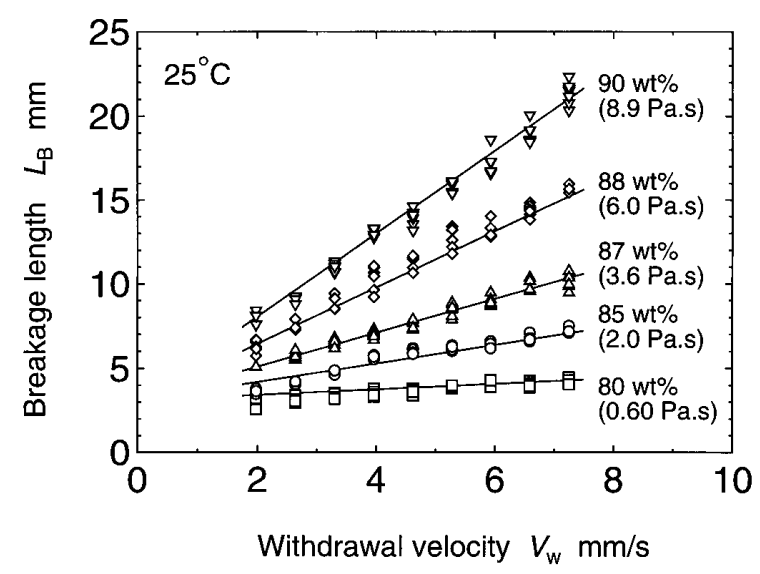

Fig.5 Breakage length dependence on withdrawal velocity in each solution of syrup. 
zero even at $\eta=0$. This phenomenon is caused by the same reason mentioned in Fig.5. The solid lines in the figure also represent the calculated results of Eq.(1). Conveniently, we can predict the breakage length in this figure if we know the shear viscosity of the coating liquid used in the dip coating and the withdrawal velocity given in the coating process. However, this experimental results are supposed to depend on the experimental conditions such as the diameter of the rod.

When we take account of the efficiency of dip coating process in the real manufacturing, the breakage time $t_{\mathrm{B}}$ is important, where $t_{\mathrm{B}}$ is defined as the time required until the filament breaks. Thus, the breakage time of filament is required as short as possible. Figure 7 shows the dependence of the breakage time on the withdrawal velocity for the Newtonian liquids. It is found that the breakage time monotonically decreases with increasing withdrawal velocity although the breakage length becomes large with increasing the withdrawal velocity as shown in Fig.5. The solid curves in the figure was calculated using the following equation:

$$
t_{\mathrm{B}}=\frac{L_{\mathrm{B}}}{V_{\mathrm{w}}}=0.28 \eta+\frac{0.0029}{V_{\mathrm{w}}}
$$

For the purpose of reducing the withdrawal time it is required to make the shear viscosity small, for example, by rise in temperature of the coating liquid in a bath, and to withdraw the rod as fast as possible.

\subsection{Results for polymeric liquids}

In this subsection, we will describe the results of the breakage length $L_{\mathrm{B}}$ for polymeric solutions. In the polymeric solutions the elongational viscosities increase with increasing

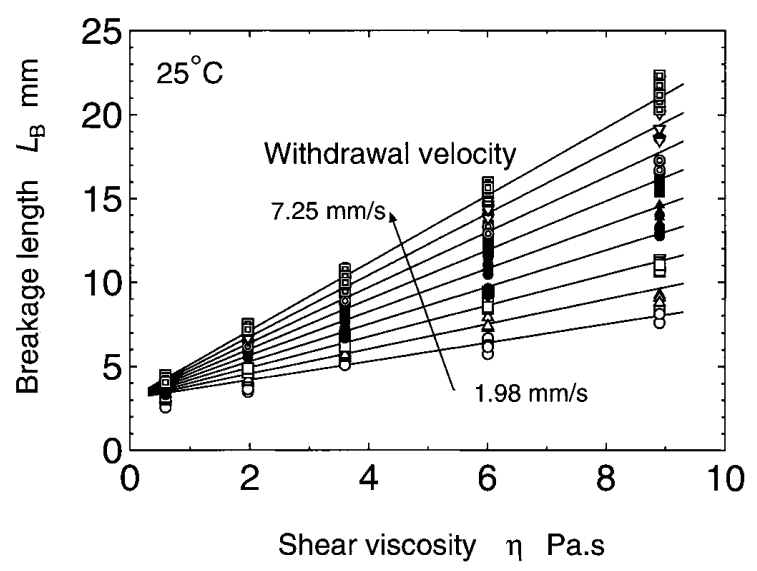

Fig.6 Breakage length dependence on shear viscosity at each withdrawal velocity. the concentration of PAA as described in the previous section. Figure 8 shows the dependence of the breakage length on the withdrawal velocity for polymeric solutions with different concentration of PAA at the fixed concentration of syrup, 85 $\mathrm{wt} \%$. The concentrations of PAA in the $85 \mathrm{wt} \%$ aqueous solution of syrup as a base liquid are $3 \mathrm{ppm}, 5 \mathrm{ppm}$ and $7 \mathrm{ppm}$. Note that three solutions have nearly the same shear viscosity. The breakage length of the polymeric solutions ranges from two to three times of that in the syrup solution in a small withdrawal velocity, that is about $2 \mathrm{~mm} / \mathrm{s}$. This increase in the breakage length seems to be caused by increase in shear viscosity of polymeric solutions at low shear rate as shown in Fig.2. However, the breakage lengths in the polymer solutions increase with increasing withdrawal velocity more remarkably compared with that in the syrup solution. The breakage length in the $7 \mathrm{ppm}$ solution of PAA, is much larger than those in the $3 \mathrm{ppm}$ and the $5 \mathrm{ppm}$ solution. This seems to be closely connected with the elongational viscosity shown in Table I, i.e., the elongational viscosity of the $7 \mathrm{ppm}$ solution is considerably larger than those in the $3 \mathrm{ppm}$ and the $5 \mathrm{ppm}$ solutions. Thus it should be noticed that a small amount of PAA, which corresponds to the concentration in ppm, causes remarkable increase in breakage length of the filament.

Figure 9 shows the dependence of the breakage time on the withdrawal velocity for various concentrations of PAA in the $85 \mathrm{wt} \%$ aqueous solution of syrup. The breakage time maintains a constant in polymeric solutions except at the small withdrawal velocity, while that of the syrup solution monotonically decreases with increasing the withdrawal velocity. Therefore, increase in withdrawal velocity is ineffective upon improvement of manufacturing efficiency in the dip coating process using polymeric solutions.

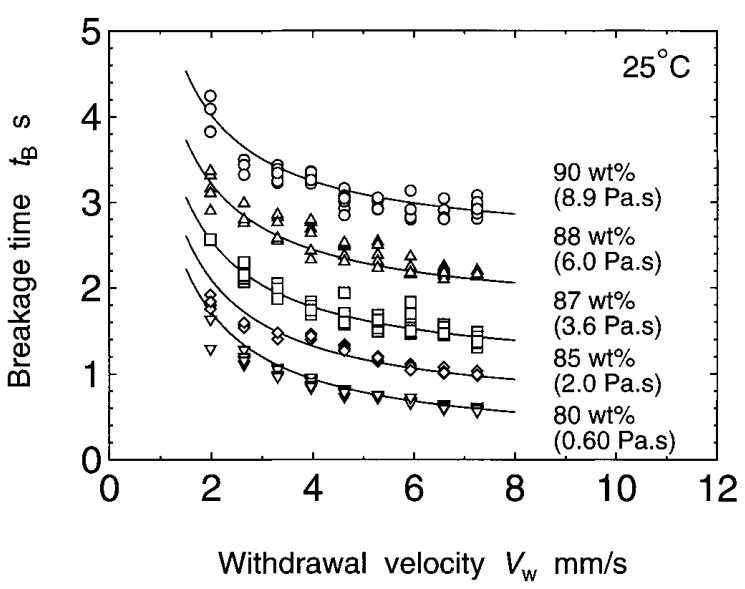

Fig.7 Breakage time dependence on withdrawal velocity in each solution of syrup. 


\section{CONCLUSION}

In the present paper, we have examined the filament formation and its breakage to attain high efficiency in coating process. We measured the filament length at breakage in withdrawing the rod from the bath using various Newtonian liquids and polymeric solutions. Consequently, it is found that it is required to make the shear viscosity small and to withdraw the rod as fast as possible in Newtonian liquids. Furthermore, we proposed the experimental equation which represents the

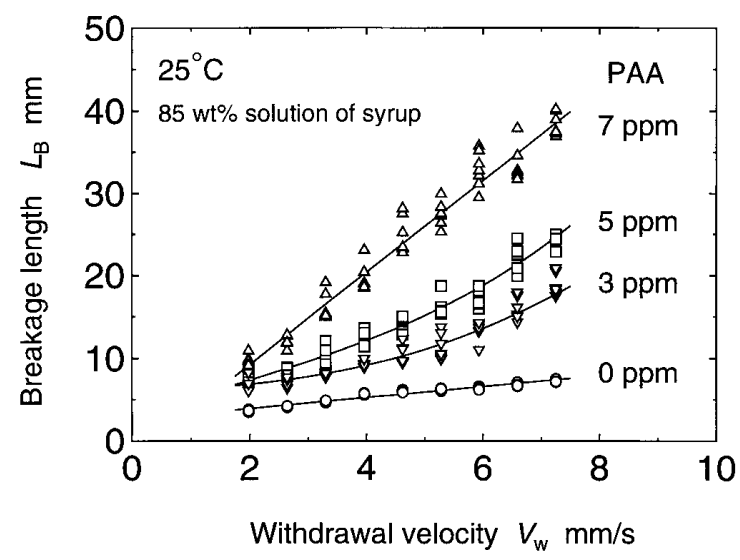

Fig.8 Breakage length dependence on withdrawal velocity in $85 \mathrm{wt} \%$ solutions of syrup containing PAA at various concentrations.

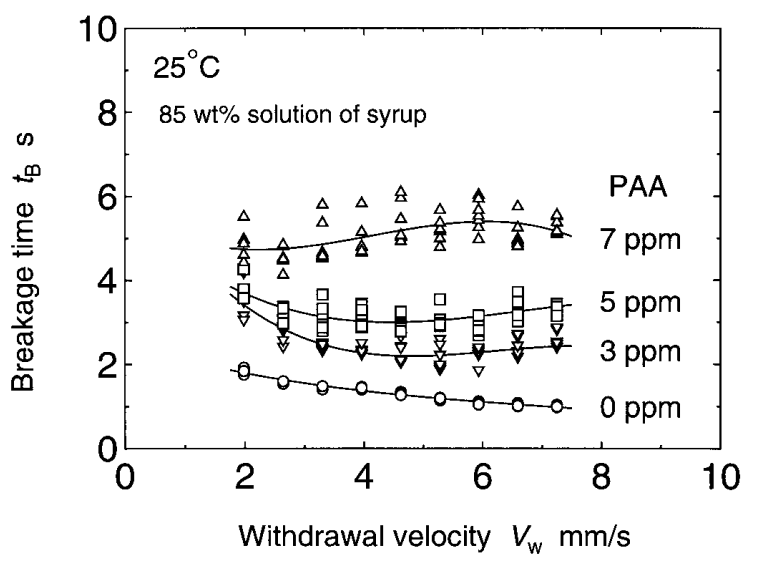

Fig.9 Breakage time dependence on withdrawal velocity in $85 \mathrm{wt} \%$ solution of syrup containing PAA at various concentrations. relation between the breakage length of the filament and the shear viscosity of the coating liquid. In the polymeric liquids, increase in withdrawal velocity is ineffective upon improvement of manufacturing efficiency in the dip coating process. The normal stress difference of the polymeric liquid, however, reduced the coating thickness ${ }^{10)}$, so that this property may be available to the thickness control of the capsule. The coating liquid containing polymer is required to have a small spinnability and a moderate normal stress difference.

When we discuss the capsule manufacturing, the coating thickness is also important. However, the coating thickness depends on many factors such as rheological properties of the liquid and the withdrawal velocity. Furthermore, the breakage length of the filament is supposed to affect the coating thickness, and a temperature dependence of the rheological properties of coating liquids on the breakage length is of importance in the real process. In the present experiment, we were not able to estimate the efficiency in coating process fixing the coating thickness and the temperature dependence. To examine the dependence of these factors on the breakage length fixing the coating thickness, the numerical simulation for coating process is available. We will consider these factors in the future.

\section{REFERENCES}

1) Ruschak KJ, Ann Rev Fluid Mech, 17, 65 (1985).

2) White DA and Tallmadge JA, Chem Eng Sci, 20, 33 (1965).

3) Spiers RP, Subbaraman CV, Wilkinson WL, Chem Eng Sci, 29, 389 (1974).

4) Marques D, Constanza V, Cerro RL, Chem Eng Sci, 33, 87 (1978).

5) Krozel JW, Palazoglu AN, Powell RL, Chem Eng Sci, 55, 3639 (2000).

6) Weinstein SJ and Ruschak KJ, Chem Eng Sci, 56, 4957 (2001).

7) Qu D, Ramé E, Garoff S, Phys Fluids, 14, 1154 (2002).

8) Tanguy P, Fortin M, Chopin L, Int J Numer Methods in Fluids, 4, 441 (1984).

9) Tanguy P, Fortin M, Chopin L, Int J Numer Methods in Fluids, 4, 459 (1984).

10) Mori N, Nojima N, Yamamoto $T$, Nakamura $\mathrm{K}, J$ Text Mach Soc Jpn -Trans-, 56, T21 (2003). (in Japanese with English abstract) 